Elect. Comm. in Probab. 13 (2008), 660-10

ELECTRONIC

COMMUNICATIONS

in PROBABILITY

\title{
SHARP INEQUALITY FOR BOUNDED SUBMARTINGALES AND THEIR DIFFERENTIAL SUBORDINATES
}

\author{
ADAM OSȨKOWSKI ${ }^{1}$ \\ Department of Mathematics, Informatics and Mechanics \\ University of Warsaw \\ Banacha 2, 02-097 Warsaw \\ Poland \\ email: ados@mimuw.edu.pl
}

Submitted July 13, 2007, accepted in final form December 8, 2008

AMS 2000 Subject classification: Primary: 60G42. Secondary: 60G46.

Keywords: Martingale, submartingale, distribution function, tail inequality, differential subordination, conditional differential subordination.

\section{Abstract}

Let $\alpha$ be a fixed number from the interval $[0,1]$. We obtain the sharp probability bounds for the maximal function of the process which is $\alpha$-differentially subordinate to a bounded submartingale. This generalizes the previous results of Burkholder and Hammack.

\section{Introduction}

Let $(\Omega, \mathscr{F}, \mathbb{P})$ be a probability space, equipped with a discrete filtration $\left(\mathscr{F}_{n}\right)$. Let $f=\left(f_{n}\right)_{n=0}^{\infty}$, $g=\left(g_{n}\right)_{n=0}^{\infty}$ be adapted integrable processes taking values in a certain separable Hilbert space $\mathscr{H}$. The difference sequences $d f=\left(d f_{n}\right), d g=\left(d g_{n}\right)$ of these processes are given by

$$
d f_{0}=f_{0}, d f_{n}=f_{n}-f_{n-1}, d g_{0}=g_{0}, d g_{n}=g_{n}-g_{n-1}, \quad n=1,2, \ldots
$$

Let $g^{*}$ stand for the maximal function of $g$, that is, $g^{*}=\max _{n}\left|g_{n}\right|$.

The following notion of differential subordination is due to Burkholder. The process $g$ is differentially subordinate to $f$ (or, in short, subordinate to $f$ ) if for any nonnegative integer $n$ we have, almost surely,

$$
\left|d g_{n}\right| \leq\left|d f_{n}\right| .
$$

We will slightly change this definition and say that $g$ is differentially subordinate to $f$, if the above inequality for the differences holds for any positive integer $n$.

Let $\alpha$ be a fixed nonnegative number. Then $g$ is $\alpha$-differentially subordinate to $f$ (or, in short, $\alpha$-subordinate to $f$ ), if it is subordinate to $f$ and for any positive integer $n$ we have

$$
\left|\mathbb{E}\left(d g_{n} \mid \mathscr{F}_{n-1}\right)\right| \leq \alpha\left|\mathbb{E}\left(d f_{n} \mid \mathscr{F}_{n-1}\right)\right| .
$$

\footnotetext{
${ }^{1}$ PARTIALLY SUPPORTED BY MEIN GRANT 1 PO3A 01229
} 
This concept was introduced by Burkholder in [2] in the special case $\alpha=1$. In general form, it first appeared in the paper by Choi [3].

In the sequel it will sometimes be convenient to work with simple processes. A process $f$ is called simple, if for any $n$ the variable $f_{n}$ is simple and there exists $N$ such that $f_{N}=f_{N+1}=f_{N+2}=\ldots$. Given such a process, we will identify it with the finite sequence $\left(f_{n}\right)_{n=0}^{N}$.

Assume that the processes $f$ and $g$ are real-valued and fix $\alpha \in[0,1]$. The objective of this paper is to establish a sharp exponential inequality for the distribution function of $g^{*}$ under the assumption that $f$ is a submartingale satisfying $\|f\|_{\infty} \leq 1$ and $g$ is $\alpha$-subordinate to $f$. To be more precise, for any $\lambda>0$ define the function $V_{\alpha, \lambda}:[-1,1] \times \mathbb{R} \rightarrow \mathbb{R}$ by the formula

$$
V_{\alpha, \lambda}\left(x_{0}, y_{0}\right)=\sup \mathbb{P}\left(g^{*} \geq \lambda\right)
$$

Here the supremum is taken over all pairs $(f, g)$ of integrable adapted processes, such that $\left(f_{0}, g_{0}\right) \equiv\left(x_{0}, y_{0}\right)$ almost surely, $f$ is a submartingale satisfying $\|f\|_{\infty} \leq 1$ and $g$ is $\alpha$-subordinate to $f$. The filtration must also vary, as well as the probability space, unless it is nonatomic. Our main result is an explicit formula for the functions $V_{\alpha, \lambda}, \lambda>0$. Usually we will omit the index $\alpha$ and write $V_{\lambda}$ instead of $V_{\alpha, \lambda}$.

Let us discuss some related results which appeared in the literature. In [1] Burkholder studied the analogous question in the case of $f, g$ being Hilbert space-valued martingales. The paper [1] contains also a related one-sided sharp exponential inequality for real martingales. This work was later extended by Hammack [4], who established a similar (two-sided) inequality under the assumption that $f$ is a submartingale bounded by 1 and $g$ is $R^{v}$-valued, $v \geq 1$, and strongly 1 -subordinate to $f$. Both papers present applications to stochastic integrals.

The paper is organized as follows. In the next section we introduce a family of special functions $U_{\lambda}, \lambda>0$ and study their properties. This enables us to establish the inequality $V_{\lambda} \leq U_{\lambda}$ in Section 3 . Then we prove the reverse inequality in the last section.

Throughout the paper, $\alpha$ is a fixed number from the interval $[0,1]$. All the considered processes are assumed to be real valued.

\section{The explicit formulas}

Let $S$ be the strip $[-1,1] \times \mathbb{R}$. Consider the following subsets of $S$ : for $0<\lambda \leq 2$,

$$
\begin{aligned}
& A_{\lambda}=\{(x, y) \in S:|y| \geq x+\lambda-1\} \\
& B_{\lambda}=\{(x, y) \in S: 1-x \leq|y|<x+\lambda-1\} \\
& C_{\lambda}=\{(x, y) \in S:|y|<1-x \text { and }|y|<x+\lambda-1\}
\end{aligned}
$$

For $\lambda \in(2,4)$, define

$$
\begin{aligned}
& A_{\lambda}=\{(x, y) \in S:|y| \geq \alpha x+\lambda-\alpha\} \\
& B_{\lambda}=\{(x, y) \in S: \alpha x+\lambda-\alpha>|y| \geq x-1+\lambda\}, \\
& C_{\lambda}=\{(x, y) \in S: x-1+\lambda>|y| \geq 1-x\} \\
& D_{\lambda}=\{(x, y) \in S: 1-x>|y| \geq-x-3+\lambda \text { and }|y|<x-1+\lambda\} \\
& E_{\lambda}=\{(x, y) \in S:-x-3+\lambda>|y|\}
\end{aligned}
$$


Finally, for $\lambda \geq 4$, let

$$
\begin{aligned}
& A_{\lambda}=\{(x, y) \in S:|y| \geq \alpha x+\lambda-\alpha\}, \\
& B_{\lambda}=\{(x, y) \in S: \alpha x+\lambda-\alpha>|y| \geq x-1+\lambda\}, \\
& C_{\lambda}=\{(x, y) \in S: x-1+\lambda>|y| \geq-x-3+\lambda\} \\
& D_{\lambda}=\{(x, y) \in S:-x-3+\lambda>|y| \geq 1-x\}, \\
& E_{\lambda}=\{(x, y) \in S: 1-x>|y|\} .
\end{aligned}
$$

Let $H: S \times(-1, \infty) \rightarrow \mathbb{R}$ be a function given by

$$
H(x, y, z)=\frac{1}{\alpha+2}\left[1+\frac{(x+1+|y|)^{1 /(\alpha+1)}((\alpha+1)(x+1)-|y|)}{(1+z)^{(\alpha+2) /(\alpha+1)}}\right]
$$

Now we will define the special functions $U_{\lambda}: S \rightarrow \mathbb{R}$. For $0<\lambda \leq 2$, let

$$
U_{\lambda}(x, y)=\left\{\begin{array}{lr}
1 & \text { if }(x, y) \in A_{\lambda}, \\
\frac{2-2 x}{1+\lambda-x-|y|} & \text { if }(x, y) \in B_{\lambda}, \\
1-\frac{(\lambda-1+x-|y|)(\lambda-1+x+|y|)}{\lambda^{2}} & \text { if }(x, y) \in C_{\lambda} .
\end{array}\right.
$$

For $2<\lambda<4$, set

$$
U_{\lambda}(x, y)= \begin{cases}1 & \text { if }(x, y) \in A_{\lambda}, \\ 1-(\alpha(x-1)-|y|+\lambda) \cdot \frac{2 \lambda-4}{\lambda^{2}} & \text { if }(x, y) \in B_{\lambda}, \\ \frac{2-2 x}{1+\lambda-x-|y|}-\frac{2(1-x)(1-\alpha)(\lambda-2)}{\lambda^{2}} & \text { if }(x, y) \in C_{\lambda}, \\ \frac{2(1-x)}{\lambda}\left[1-\frac{(1-\alpha)(\lambda-2)}{\lambda}\right]-\frac{(1-x)^{2}-|y|^{2}}{\lambda^{2}} & \text { if }(x, y) \in D_{\lambda}, \\ a_{\lambda} H(x, y, \lambda-3)+b_{\lambda} & \text { if }(x, y) \in E_{\lambda},\end{cases}
$$

where

$$
a_{\lambda}=-\frac{2(1+\alpha)(\lambda-2)^{2}}{\lambda^{2}}, \quad b_{\lambda}=1-\frac{4(\lambda-2)(1-\alpha)}{\lambda^{2}} .
$$

For $\lambda \geq 4$, set

$$
U_{\lambda}(x, y)= \begin{cases}1 & \text { if }(x, y) \in A_{\lambda}, \\ 1-\frac{\alpha(x-1)-|y|+\lambda}{4} & \text { if }(x, y) \in B_{\lambda}, \\ \frac{2-2 x}{1+\lambda-x-|y|}-\frac{(1-x)(1-\alpha)}{4} & \text { if }(x, y) \in C_{\lambda}, \\ \frac{(1-x)(1+\alpha)}{4} \exp \left(\frac{3+x+|y|-\lambda}{2(\alpha+1)}\right) & \text { if }(x, y) \in D_{\lambda}, \\ a_{\lambda} H(x, y, 1)+b_{\lambda} & \text { if }(x, y) \in E_{\lambda},\end{cases}
$$

where

$$
a_{\lambda}=-b_{\lambda}=-\frac{(1+\alpha)}{2} \exp \left(\frac{4-\lambda}{2 \alpha+2}\right) \text {. }
$$

For $\alpha=1$, the formulas (3), (4), (6) give the special functions constructed by Hammack [4]. The key properties of $U_{\lambda}$ are described in the two lemmas below.

Lemma 1. For $\lambda>2$, let $\phi_{\lambda}, \psi_{\lambda}$ denote the partial derivatives of $U_{\lambda}$ with respect to $x$, $y$ on the interiors of $A_{\lambda}, B_{\lambda}, C_{\lambda}, D_{\lambda}, E_{\lambda}$, extended continuously to the whole of these sets. The following statements hold. 
(i) The functions $U_{\lambda}, \lambda>2$, are continuous on $S \backslash\{(1, \pm \lambda)\}$.

(ii) Let

$$
S_{\lambda}=\{(x, y) \in[-1,1] \times \mathbb{R}:|y| \neq \alpha x+\lambda-\alpha \text { and }|y| \neq x+\lambda-1\} .
$$

Then

$$
\phi_{\lambda}, \psi_{\lambda}, \lambda>2 \text {, are continuous on } S_{\lambda} .
$$

(iii) For any $(x, y) \in S$, the function $\lambda \mapsto U_{\lambda}(x, y), \lambda>0$, is left-continuous.

(iv) For any $\lambda>2$ we have the inequality

$$
\phi_{\lambda} \leq-\alpha\left|\psi_{\lambda}\right|
$$

(v) For $\lambda>2$ and any $(x, y) \in S$ we have $\chi_{\{|y| \geq \lambda\}} \leq U_{\lambda}(x, y) \leq 1$.

Proof. We start with computing the derivatives. Let $y^{\prime}=y /|y|$ stand for the sign of $y$, with $y^{\prime}=0$ if $y=0$. For $\lambda \in(2,4)$ we have

$$
\begin{aligned}
& \phi_{\lambda}(x, y)= \begin{cases}0 & \text { if }(x, y) \in A_{\lambda}, \\
-\frac{(2 \lambda-4) \alpha}{\lambda^{2}} & \text { if }(x, y) \in B_{\lambda}, \\
-\frac{2 \lambda-2|y|}{(1+\lambda-x-|y|)^{2}}+\frac{(2 \lambda-4)(1-\alpha)}{\lambda^{2}} & \text { if }(x, y) \in C_{\lambda}, \\
-\frac{2}{\lambda}\left[1-\frac{(1-\alpha)(\lambda-2)}{\lambda}\right]+\frac{2(1-x)}{\lambda^{2}} & \text { if }(x, y) \in D_{\lambda}, \\
-c_{\lambda}(x+|y|+1)^{-\alpha /(\alpha+1)}\left(x+1+\frac{\alpha}{\alpha+1}|y|\right) & \text { if }(x, y) \in E_{\lambda},\end{cases} \\
& \psi_{\lambda}(x, y)= \begin{cases}0 & \text { if }(x, y) \in A_{\lambda}, \\
\frac{2 \lambda-4}{\lambda^{2}} y^{\prime} & \text { if }(x, y) \in B_{\lambda}, \\
\frac{2-2 x}{(1+\lambda-x-|y|)^{2}} y^{\prime} & \text { if }(x, y) \in C_{\lambda}, \\
\frac{2 y}{\lambda^{2}} & \text { if }(x, y) \in D_{\lambda}, \\
c_{\lambda}(x+|y|+1)^{-\alpha /(\alpha+1)} \frac{y}{1+\alpha} & \text { if }(x, y) \in E_{\lambda},\end{cases}
\end{aligned}
$$

where

$$
c_{\lambda}=2(1+\alpha)(\lambda-2)^{\alpha /(\alpha+1)} \lambda^{-2} .
$$

Finally, for $\lambda \geq 4$, set

$$
\begin{gathered}
\phi_{\lambda}(x, y)= \begin{cases}0 & \text { if }(x, y) \in A_{\lambda}, \\
-\frac{\alpha}{4} \frac{2 \lambda-2|y|}{(1+\lambda-x|y|)^{2}}+\frac{1-\alpha}{4} & \text { if }(x, y) \in B_{\lambda}, \\
-\frac{x+1+2 \alpha}{8} \exp \left(\frac{x+|y|+3-\lambda}{2(\alpha+1)}\right) & \text { if }(x, y) \in C_{\lambda}, \\
-c_{\lambda}(x+|y|+1)^{-\alpha /(\alpha+1)}\left(x+1+\frac{\alpha}{\alpha+1}|y|\right) & \text { if }(x, y) \in D_{\lambda},\end{cases} \\
\psi_{\lambda}(x, y)= \begin{cases}0 & \text { if }(x, y) \in E_{\lambda}, \\
\frac{1}{4} y^{\prime} & \text { if }(x, y) \in A_{\lambda}, \\
\frac{2-2 x}{(1+\lambda-x-|y|)^{2}} y^{\prime} & \text { if }(x, y) \in B_{\lambda}, \\
\frac{(1-x)}{8} \exp \left(\frac{x+|y|+3-\lambda}{2(\alpha+1)}\right) y^{\prime} & \text { if }(x, y) \in C_{\lambda}, \\
c_{\lambda}(x+|y|+1)^{-\alpha /(\alpha+1)} \frac{y}{1+\alpha} & \text { if }(x, y) \in D_{\lambda},\end{cases} \\
\end{gathered}
$$


where

$$
c_{\lambda}=(1+\alpha) 2^{-(2 \alpha+3) /(\alpha+1)} \exp \left(\frac{4-\lambda}{2(\alpha+1)}\right) .
$$

Now the properties (i), (ii), (iii) follow by straightforward computation. To prove (iv), note first that for any $\lambda>2$ the condition (9) is clearly satisfied on the sets $A_{\lambda}$ and $B_{\lambda}$. Suppose $(x, y) \in C_{\lambda}$. Then $\lambda-|y| \in[0,4], 1-x \leq \min \{\lambda-|y|, 4-\lambda+|y|\}$ and (9) takes form

$$
-2(\lambda-|y|)+\frac{2 \lambda-4}{\lambda^{2}}(1-\alpha)(1-x+\lambda-|y|)^{2}+2 \alpha(1-x) \leq 0,
$$

or

$$
-2(\lambda-|y|)+\frac{1-\alpha}{4} \cdot(1-x+\lambda-|y|)^{2}+2 \alpha(1-x) \leq 0,
$$

depending on whether $\lambda<4$ or $\lambda \geq 4$. As $(2 \lambda-4) / \lambda^{2} \leq \frac{1}{4}$, it suffices to show (10). If $\lambda-|y| \leq 2$, then, as $1-x \leq \lambda-|y|$, the left-hand side does not exceed

$$
\begin{aligned}
-2(\lambda-|y|)+(1-\alpha)(\lambda-|y|)^{2}+2 \alpha(\lambda-|y|) & =(\lambda-|y|)(-2+(1-\alpha)(\lambda-|y|)+2 \alpha) \\
& \leq(\lambda-|y|)(-2+2(1-\alpha)+2 \alpha)=0 .
\end{aligned}
$$

Similarly, if $\lambda-|y| \in(2,4]$, then we use the bound $1-x \leq 4-\lambda+|y|$ and conclude that the left-hand side of (10) is not greater than

$$
-2(\lambda-|y|)+4(1-\alpha)+2 \alpha(4-\lambda+|y|)=-2(\lambda-|y|-2)(1+\alpha) \leq 0
$$

and we are done with the case $(x, y) \in C_{\lambda}$.

Assume that $(x, y) \in D_{\lambda}$. For $\lambda \in(2,4)$, the inequality (9) is equivalent to

$$
-\frac{2}{\lambda}\left[1-\frac{(1-\alpha)(\lambda-2)}{\lambda}\right]+\frac{2-2 x}{\lambda^{2}} \leq-\frac{2 \alpha|y|}{\lambda^{2}}
$$

or, after some simplifications, $\alpha|y|+1-x \leq 2+\alpha \lambda-2 \alpha$. It is easy to check that $\alpha|y|+1-x$ attains its maximum for $x=-1$ and $|y|=\lambda-2$ and then we have the equality. If $(x, y) \in D_{\lambda}$ and $\lambda \geq 4$, then (9) takes form $-(2 \alpha+1+x) \leq-\alpha(1-x)$, or $(x+1)(\alpha+1) \geq 0$. Finally, on the set $E_{\lambda}$, the inequality (9) is obvious.

(v) By (9), we have $\phi_{\lambda} \leq 0$, so $U_{\lambda}(x, y) \geq U_{\lambda}(1, y)=\chi_{\{|y| \geq \lambda\}}$. Furthermore, as $U_{\lambda}(x, y)=1$ for $|y| \geq \lambda$ and $\psi_{\lambda}(x, y) y^{\prime} \geq 0$ on $S_{\lambda}$, the second estimate follows.

Lemma 2. Let $x, h, y, k$ be fixed real numbers, satisfying $x, x+h \in[-1,1]$ and $|k| \leq|h|$. Then for any $\lambda>2$ and $\alpha \in[0,1)$,

$$
U_{\lambda}(x+h, y+k) \leq U_{\lambda}(x, y)+\phi_{\lambda}(x, y) h+\psi_{\lambda}(x, y) k
$$

We will need the following fact, proved by Burkholder; see page 17 of [1].

Lemma 3. Let $x, h, y, k, z$ be real numbers satisfying $|k| \leq|h|$ and $z>-1$. Then the function

$$
F(t)=H(x+t h, y+t k, z),
$$

defined on $\{t:|x+t h| \leq 1\}$, is convex. 
Proof of the Lemma 2. Consider the function

$$
G(t)=G_{x, y, h, k}(t)=U_{\lambda}(x+t h, y+t k),
$$

defined on the set $\{t:|x+t h| \leq 1\}$. It is easy to check that $G$ is continuous. As explained in [1], the inequality (11) follows once the concavity of $G$ is established. This will be done by proving the inequality $G^{\prime \prime} \leq 0$ at the points, where $G$ is twice differentiable and checking the inequality $G_{+}^{\prime}(t) \leq G_{-}^{\prime}(t)$ for those $t$, for which $G$ is not differentiable (even once). Note that we may assume $t=0$, by a translation argument $G_{x, y, h, k}^{\prime \prime}(t)=G_{x+t h, y+t k, h, k}^{\prime \prime}(0)$, with analogous equalities for onesided derivatives. Clearly, we may assume that $h \geq 0$, changing the signs of both $h, k$, if necessary. Due to the symmetry of $U_{\lambda}$, we are allowed to consider $y \geq 0$ only.

We start from the observation that $G^{\prime \prime}(0)=0$ on the interior of $A_{\lambda}$ and $G_{+}^{\prime}(0) \leq G_{-}^{\prime}(0)$ for $(x, y) \in$ $A_{\lambda} \cap \bar{B}_{\lambda}$. The latter inequality holds since $U_{\lambda} \equiv 1$ on $A_{\lambda}$ and $U_{\lambda} \leq 1$ on $B_{\lambda}$. For the remaining inequalities, we consider the cases $\lambda \in(2,4), \lambda \geq 4$ separately.

The case $\lambda \in(2,4)$. The inequality $G^{\prime \prime}(0) \leq 0$ is clear for $(x, y)$ lying in the interior of $B_{\lambda}$. On $C_{\lambda}$, we have

$$
G^{\prime \prime}(0)=-\frac{4(h+k)(h(\lambda-y)-k(1-x))}{(1-x-y+\lambda)^{3}} \leq 0,
$$

which follows from $|k| \leq h$ and the fact that $\lambda-y \geq 1-x$. For $(x, y)$ in the interior of $D_{\lambda}$,

$$
G^{\prime \prime}(0)=\frac{-h^{2}+k^{2}}{\lambda^{2}} \leq 0,
$$

as $|k| \leq h$. Finally, on $E_{\lambda}$, the concavity follows by Lemma 3 .

It remains to check the inequalities for one-sided derivatives. By Lemma 1 (ii), the points $(x, y)$, for which $G$ is not differentiable at 0 , do not belong to $S_{\lambda}$. Since we excluded the set $A_{\lambda} \cap \bar{B}_{\lambda}$, they lie on the line $y=x-1+\lambda$. For such points $(x, y)$, the left derivative equals

$$
G_{-}^{\prime}(0)=-\frac{2 \lambda-4}{\lambda^{2}}(\alpha h-k)
$$

while the right one is given by

$$
G_{+}^{\prime}(0)=\frac{-h+k}{2(\lambda-y)}+\frac{(2 \lambda-4)(1-\alpha) h}{\lambda^{2}},
$$

or

$$
G_{+}^{\prime}(0)=-\frac{2 h}{\lambda}\left[1-\frac{(1-\alpha)(\lambda-2)}{\lambda}\right]+\frac{2(1-x) h+2 y k}{\lambda^{2}},
$$

depending on whether $y \geq 1-x$ or $y<1-x$. In the first case, the inequality $G_{+}^{\prime}(0) \leq G_{-}^{\prime}(0)$ reduces to

$$
(h-k)\left(\frac{1}{2(\lambda-y)}-\frac{2(\lambda-2)}{\lambda^{2}}\right) \geq 0,
$$

while in the remaining one,

$$
\frac{2}{\lambda^{2}}(h-k)(y-(\lambda-2)) \geq 0
$$

Both inequalities follow from the estimate $\lambda-y \leq 2$ and the condition $|k| \leq h$. 
The case $\lambda \geq 4$. On the set $B_{\lambda}$ the concavity is clear. For $C_{\lambda}$, we have that the formula (12) holds. If $(x, y)$ lies in the interior of $D_{\lambda}$, then

$$
G^{\prime \prime}(0)=\frac{1}{8} \exp \left(\frac{3+x+y-\lambda}{2(\alpha+1)}\right)\left[\frac{1-x}{2(\alpha+1)} \cdot\left(-h^{2}+k^{2}\right)-\left(2-\frac{1-x}{\alpha+1}\right)\left(h^{2}+h k\right)\right] \leq 0,
$$

since $|k| \leq h$ and $(1-x) /(\alpha+1) \leq 2$. The concavity on $E_{\lambda}$ is a consequence of Lemma 3. It remains to check the inequality for one-sided derivatives. By Lemma 1 (ii), we may assume $y=x+\lambda-1$, and the inequality $G_{+}^{\prime}(0) \leq G_{-}^{\prime}(0)$ reads

$$
\frac{1}{2}(h-k)\left(\frac{1}{\lambda-y}-\frac{1}{2}\right) \geq 0
$$

an obvious one, as $\lambda-y \leq 2$.

\section{The main theorem}

Now we may state and prove the main result of the paper.

Theorem 1. Suppose $f$ is a submartingale satisfying $\|f\|_{\infty} \leq 1$ and $g$ is an adapted process which is $\alpha$-subordinate to $f$. Then for all $\lambda>0$ we have

$$
\mathbb{P}\left(g^{*} \geq \lambda\right) \leq \mathbb{E} U_{\lambda}\left(f_{0}, g_{0}\right) .
$$

Proof. If $\lambda \leq 2$, then this follows immediately from the result of Hammack [4]; indeed, note that $U_{\lambda}$ coincides with Hammack's special function and, furthermore, since $g$ is $\alpha$-subordinate to $f$, it is also 1-subordinate to $f$.

Fix $\lambda>2$. We may assume $\alpha<1$. It suffices to show that for any nonnegative integer $n$,

$$
\mathbb{P}\left(\left|g_{n}\right| \geq \lambda\right) \leq \mathbb{E} U_{\lambda}\left(f_{0}, g_{0}\right) .
$$

To see that this implies (13), fix $\varepsilon>0$ and consider a stopping time $\tau=\inf \left\{k:\left|g_{k}\right| \geq \lambda-\varepsilon\right\}$. The process $f^{\tau}=\left(f_{\tau \wedge n}\right)$, by Doob's optional sampling theorem, is a submartingale. Furthermore, we obviously have that $\left\|f^{\tau}\right\|_{\infty} \leq 1$ and the process $g^{\tau}=\left(g_{\tau \wedge n}\right)$ is $\alpha$-subordinate to $f^{\tau}$. Therefore, by (14),

$$
\mathbb{P}\left(\left|g_{n}^{\tau}\right| \geq \lambda-\varepsilon\right) \leq \mathbb{E} U_{\lambda-\varepsilon}\left(f_{0}^{\tau}, g_{0}^{\tau}\right)=\mathbb{E} U_{\lambda-\varepsilon}\left(f_{0}, g_{0}\right) .
$$

Now if we let $n \rightarrow \infty$, we obtain $\mathbb{P}\left(g^{*} \geq \lambda\right) \leq \mathbb{E} U_{\lambda-\varepsilon}\left(f_{0}, g_{0}\right)$ and by left-continuity of $U_{\lambda}$ as a function of $\lambda$, (13) follows.

Thus it remains to establish (14). By Lemma 1 (v), $\mathbb{P}\left(\left|g_{n}\right| \geq \lambda\right) \leq \mathbb{E} U_{\lambda}\left(f_{n}, g_{n}\right)$ and it suffices to show that for all $1 \leq j \leq n$ we have

$$
\mathbb{E} U_{\lambda}\left(f_{j}, g_{j}\right) \leq \mathbb{E} U_{\lambda}\left(f_{j-1}, g_{j-1}\right) .
$$

To do this, note that, since $\left|d g_{j}\right| \leq\left|d f_{j}\right|$ almost surely, the inequality (11) yields

$$
U_{\lambda}\left(f_{j}, g_{j}\right) \leq U_{\lambda}\left(f_{j-1}, g_{j-1}\right)+\phi_{\lambda}\left(f_{j-1}, g_{j-1}\right) d f_{j}+\psi_{\lambda}\left(f_{j-1}, g_{j-1}\right) d g_{j}
$$

with probability 1 . Assume for now that $\phi_{\lambda}\left(f_{j-1}, g_{j-1}\right) d f_{j}, \psi_{\lambda}\left(f_{j-1}, g_{j-1}\right) d g_{j}$ are integrable. By $\alpha$-subordination, the condition (9) and the submartingale property $\mathbb{E}\left(d_{j} \mid \mathscr{F}_{j-1}\right) \geq 0$, we have

$$
\mathbb{E}\left[\phi_{\lambda}\left(f_{j-1}, g_{j-1}\right) d f_{j}+\psi_{\lambda}\left(f_{j-1}, g_{j-1}\right) d g_{j} \mid \mathscr{F}_{j-1}\right]
$$




$$
\begin{aligned}
& \leq \phi_{\lambda}\left(f_{j-1}, g_{j-1}\right) \mathbb{E}\left(d f_{j} \mid \mathscr{F}_{j-1}\right)+\left|\psi_{\lambda}\left(f_{j-1}, g_{j-1}\right)\right| \cdot\left|\mathbb{E}\left(d g_{j} \mid \mathscr{F}_{j-1}\right)\right| \\
& \leq\left[\phi_{\lambda}\left(f_{j-1}, g_{j-1}\right)+\alpha\left|\psi_{\lambda}\left(f_{j-1}, g_{j-1}\right)\right|\right] \mathbb{E}\left(d f_{j} \mid \mathscr{F}_{j-1}\right) \leq 0 .
\end{aligned}
$$

Therefore, it suffices to take the expectation of both sides of (16) to obtain (15).

Thus we will be done if we show the integrability of $\phi_{\lambda}\left(f_{j-1}, g_{j-1}\right) d f_{j}$ and $\psi_{\lambda}\left(f_{j-1}, g_{j-1}\right) d g_{j}$. In both the cases $\lambda \in(2,4), \lambda \geq 4$, all we need is that the variables

$$
\frac{2 \lambda-2\left|g_{j-1}\right|}{\left(1-f_{j-1}-\left|g_{j-1}\right|+\lambda\right)^{2}} d f_{j} \text { and } \frac{2-2 f_{j-1}}{\left(1-f_{j-1}-\left|g_{j-1}\right|+\lambda\right)^{2}} d g_{j}
$$

are integrable on the set $K=\left\{\left|g_{j-1}\right|<f_{j-1}+\lambda-1,\left|g_{j-1}\right| \geq \lambda-1\right\}$, since outside it the derivatives $\phi_{\lambda}, \psi_{\lambda}$ are bounded by a constant depending only on $\alpha, \lambda$ and $\left|d f_{j}\right|,\left|d g_{j}\right|$ do not exceed 2 . The integrability is proved exactly in the same manner as in [4]. We omit the details.

We will now establish the following sharp exponential inequality.

Theorem 2. Suppose $f$ is a submartingale satisfying $\|f\|_{\infty} \leq 1$ and $g$ is an adapted process which is $\alpha$-subordinate to $f$. In addition, assume that $\left|g_{0}\right| \leq\left|f_{0}\right|$ with probability 1 . Then for $\lambda \geq 4$ we have

$$
\mathbb{P}\left(g^{*} \geq \lambda\right) \leq \gamma e^{-\lambda /(2 \alpha+2)},
$$

where

$$
\gamma=\frac{1+\alpha}{2 \alpha+4}\left(\alpha+1+2^{-\frac{\alpha+2}{\alpha+1}}\right) \exp \left(\frac{2}{\alpha+1}\right) .
$$

The inequality is sharp.

This should be compared to Burkholder's estimate (Theorem 8.1 in [1])

$$
\mathbb{P}\left(g^{*} \geq \lambda\right) \leq \frac{e^{2}}{4} \cdot e^{-\lambda}, \quad \lambda \geq 2,
$$

in the case when $f, g$ are Hilbert space-valued martingales and $g$ is subordinate to $f$. For $\alpha=1$, we obtain the inequality of Hammack [4],

$$
\mathbb{P}\left(g^{*} \geq \lambda\right) \leq \frac{(8+\sqrt{2}) e}{12} \cdot e^{-\lambda / 4}, \quad \lambda \geq 4 .
$$

Proof of the inequality (18). We will prove that the maximum of $U_{\lambda}$ on the set $K=\{(x, y) \in S$ : $|y| \leq|x|\}$ is given by the right hand side of (18). This, together with the inequality (13) and the assumption $\mathbb{P}\left(\left(f_{0}, g_{0}\right) \in K\right)=1$, will imply the desired estimate. Clearly, by symmetry, we may restrict ourselves to the set $K^{+}=K \cap\{y \geq 0\}$. If $(x, y) \in K^{+}$and $x \geq 0$, then it is easy to check that

$$
U_{\lambda}(x, y) \leq U_{\lambda}((x+y) / 2,(x+y) / 2) .
$$

Furthermore, a straightforward computation shows that the function $F:[0,1] \rightarrow \mathbb{R}$ given by $F(s)=U_{\lambda}(s, s)$ is nonincreasing. Thus we have $U_{\lambda}(x, y) \leq U_{\lambda}(0,0)$. On the other hand, if $(x, y) \in$ $K^{+}$and $x \leq 0$, then it is easy to prove that $U_{\lambda}(x, y) \leq U_{\lambda}(-1, x+y+1)$ and the function $G:[0,1] \rightarrow \mathbb{R}$ given by $G(s)=U_{\lambda}(-1, s)$ is nondecreasing. Combining all these facts we have that for any $(x, y) \in K^{+}$,

$$
U_{\lambda}(x, y) \leq U_{\lambda}(-1,1)=\gamma e^{-\lambda /(2 \alpha+2)} .
$$

Thus (18) holds. The sharpness will be shown in the next section. 


\section{Sharpness}

Recall the function $V_{\lambda}=V_{\alpha, \lambda}$ defined by (1) in the introduction. The main result in this section is Theorem 3 below, which, combined with Theorem 1 , implies that the functions $U_{\lambda}$ and $V_{\lambda}$ coincide. If we apply this at the point $(-1,1)$ and use the equality appearing in (19), we obtain that the inequality (18) is sharp.

Theorem 3. For any $\lambda>0$ we have

$$
U_{\lambda} \leq V_{\lambda}
$$

The main tool in the proof is the following "splicing" argument. Assume that the underlying probability space is the interval $[0,1]$ with the Lebesgue measure.

Lemma 4. Fix $\left(x_{0}, y_{0}\right) \in[-1,1] \times \mathbb{R}$. Suppose there exists a filtration and a pair $(f, g)$ of simple adapted processes, starting from $\left(x_{0}, y_{0}\right)$, such that $f$ is a submartingale satisfying $\|f\|_{\infty} \leq 1$ and $g$ is $\alpha$-subordinate to $f$. Then $V_{\lambda}\left(x_{0}, y_{0}\right) \geq \mathbb{E} V_{\lambda}\left(f_{\infty}, g_{\infty}\right)$ for $\lambda>0$.

Proof. Let $N$ be such that $\left(f_{N}, g_{N}\right)=\left(f_{\infty}, g_{\infty}\right)$ and fix $\varepsilon>0$. With no loss of generality, we may assume that $\sigma$-field generated by $f, g$ is generated by the family of intervals $\left\{\left[a_{i}, a_{i+1}\right)\right.$ : $i=1,2, \ldots, M-1\}, 0=a_{1}<a_{2}<\ldots<a_{M}=1$. For any $i \in\{1,2, \ldots, M-1\}$, denote $x_{0}^{i}=f_{N}\left(a_{i}\right), y_{0}^{i}=g_{N}\left(a_{i}\right)$. There exists a filtration and a pair $\left(f^{i}, g^{i}\right)$ of adapted processes, with $f$ being a submartingale bounded in absolute value by 1 and $g$ being $\alpha$-subordinate to $f$, which satisfy $f_{0}^{i}=x_{0}^{i} \chi_{[0,1)}, g_{0}^{i}=y_{0}^{i} \chi_{[0,1)}$ and $\mathbb{P}\left(\left(g^{i}\right)^{*} \geq \lambda\right)>\mathbb{E} V_{\lambda}\left(f_{0}^{i}, g_{0}^{i}\right)-\varepsilon$. Define the processes $F, G$ by $F_{k}=f_{k}, G_{k}=g_{k}$ if $k \leq N$ and

$$
\begin{aligned}
& F_{k}(\omega)=\sum_{i=1}^{M-1} f_{k-N}^{i}\left(\left(\omega-a_{i}\right) /\left(a_{i+1}-a_{i}\right)\right) \chi_{\left[a_{i}, a_{i+1}\right)}(\omega), \\
& G_{k}(\omega)=\sum_{i=1}^{M-1} g_{k-N}^{i}\left(\left(\omega-a_{i}\right) /\left(a_{i+1}-a_{i}\right)\right) \chi_{\left[a_{i}, a_{i+1}\right)}(\omega)
\end{aligned}
$$

for $k>N$. It is easy to check that there exists a filtration, relative to which the process $F$ is a submartingale satisfying $\|F\|_{\infty} \leq 1$ and $G$ is an adapted process which is $\alpha$-subordinate to $F$. Furthermore, we have

$$
\begin{aligned}
\mathbb{P}\left(G^{*} \geq \lambda\right) \geq \sum_{i=1}^{M-1}\left(a_{i+1}-a_{i}\right) \mathbb{P}\left(\left(g^{i}\right)^{*} \geq \lambda\right) \\
\quad>\sum_{i=1}^{M-1}\left(a_{i+1}-a_{i}\right)\left(\mathbb{E} V_{\lambda}\left(f_{0}^{i}, g_{0}^{i}\right)-\varepsilon\right)=\mathbb{E} V_{\lambda}\left(f_{\infty}, g_{\infty}\right)-\varepsilon
\end{aligned}
$$

Since $\varepsilon$ was arbitrary, the result follows.

Proof of Theorem 3. First note the following obvious properties of the functions $V_{\lambda}, \lambda>0$ : we have $V_{\lambda} \in[0,1]$ and $V_{\lambda}(x, y)=V_{\lambda}(x,-y)$. The second equality is an immediate consequence of the fact that if $g$ is $\alpha$-subordinate to $f$, then so is $-g$.

In the proof of Theorem 3 we repeat several times the following procedure. Having fixed a point $\left(x_{0}, y_{0}\right)$ from the strip $S$, we construct certain simple finite processes $f, g$ starting from $\left(x_{0}, y_{0}\right)$, take their natural filtration $\left(\mathscr{F}_{n}\right)$, apply Lemma 4 and thus obtain a bound for $V_{\lambda}\left(x_{0}, y_{0}\right)$. All the constructed processes appearing in the proof below are easily checked to satisfy the conditions 
of this lemma: the condition $\|f\|_{\infty} \leq 1$ is straightforward, while the $\alpha$-subordination and the fact that $f$ is a submartingale are implied by the following. For any $n \geq 1$, either $d f_{n}$ satisfies $\mathbb{E}\left(d f_{n} \mid \mathscr{F}_{n-1}\right)=0$ and $d g_{n}= \pm d f_{n}$, or $d f_{n} \geq 0$ and $d g_{n}= \pm \alpha d f_{n}$.

We will consider the cases $\lambda \leq 2,2<\lambda<4, \lambda \geq 4$ separately. Note that by symmetry, it suffices to establish (20) on $S \cap\{y \geq 0\}$.

The case $\lambda \leq 2$. Assume $\left(x_{0}, y_{0}\right) \in A_{\lambda}$. If $y_{0} \geq \lambda$, then $g^{*} \geq \lambda$ almost surely, so $V_{\lambda}\left(x_{0}, y_{0}\right) \geq 1=$ $U_{\lambda}\left(x_{0}, y_{0}\right)$. If $\lambda>y_{0} \geq \alpha x_{0}-\alpha+\lambda$, then let $\left(f_{0}, g_{0}\right) \equiv\left(x_{0}, y_{0}\right)$,

$$
d f_{1}=\left(1-x_{0}\right) \chi_{[0,1]} \text { and } d g_{1}=\alpha d f_{1} .
$$

Then we have $g_{1}=y_{0}+\alpha-\alpha x_{0} \geq \lambda$, which implies $g^{*} \geq \lambda$ almost surely and (20) follows. Now suppose $\left(x_{0}, y_{0}\right) \in A_{\lambda}$ and $y_{0}<\alpha x_{0}-\alpha+\lambda$. Let $(f, g) \equiv\left(x_{0}, y_{0}\right)$,

$$
d f_{1}=\frac{y_{0}-x_{0}+1-\lambda}{1-\alpha} \chi_{[0,1]}, d g_{1}=\alpha d f_{1}
$$

and

$$
d f_{2}=d g_{2}=\beta \chi_{[0,1-\beta / 2)}+(\beta-2) \chi_{[1-\beta / 2,1]}
$$

where

$$
\beta=\frac{\alpha x_{0}-y_{0}-\alpha+\lambda}{1-\alpha} \in[0,2] .
$$

Then $\left(f_{2}, g_{2}\right)$ takes values $(-1, \lambda-2),(1, \lambda)$ with probabilities $\beta / 2,1-\beta / 2$, respectively, so, by Lemma 4 ,

$$
V_{\lambda}\left(x_{0}, y_{0}\right) \geq \frac{\beta}{2} V_{\lambda}(-1, \lambda-2)+\left(1-\frac{\beta}{2}\right) V_{\lambda}(1, \lambda)=\frac{\beta}{2} V_{\lambda}(-1,2-\lambda)+1-\frac{\beta}{2} .
$$

Note that $(-1,2-\lambda) \in A_{\lambda}$. If $2-\lambda \geq \alpha \cdot(-1)-\alpha+\lambda$, then, as already proved, $V_{\lambda}(-1,2-\lambda)=1$ and $V_{\lambda}\left(x_{0}, y_{0}\right) \geq 1=U_{\lambda}\left(x_{0}, y_{0}\right)$. If the converse inequality holds, i.e., $2-\lambda<-2 \alpha+\lambda$, then we may apply (25) to $x_{0}=-1, y_{0}=2-\lambda$ to get

$$
V_{\lambda}(-1,2-\lambda) \geq \frac{\beta}{2} V_{\lambda}(-1,2-\lambda)+1-\frac{\beta}{2},
$$

or $V_{\lambda}(-1,2-\lambda) \geq 1$. Thus we established $V_{\lambda}\left(x_{0}, y_{0}\right)=1$ for any $\left(x_{0}, y_{0}\right) \in A_{\lambda}$.

Suppose then, that $\left(x_{0}, y_{0}\right) \in B_{\lambda}$. Let

$$
\beta=\frac{2\left(1-x_{0}\right)}{1-x_{0}-y_{0}+\lambda} \in[0,1]
$$

and consider a pair $(f, g)$ starting from $\left(x_{0}, y_{0}\right)$ and satisfying

$$
d f_{1}=-d g_{1}=-\frac{x_{0}-y_{0}-1+\lambda}{2} \chi_{[0, \beta)}+\left(1-x_{0}\right) \chi_{[\beta, 1]} .
$$

On $[0, \beta)$, the pair $\left(f_{1}, g_{1}\right)$ lies in $A_{\lambda}$; Lemma 4 implies $V_{\lambda}\left(x_{0}, y_{0}\right) \geq \beta=U_{\lambda}\left(x_{0}, y_{0}\right)$.

Finally, for $\left(x_{0}, y_{0}\right) \in C_{\lambda}$, let $(f, g)$ start from $\left(x_{0}, y_{0}\right)$ and

$$
d f_{1}=-d g_{1}=\frac{-x_{0}-\lambda+1+y_{0}}{2} \chi_{[0, \gamma)}+\frac{y_{0}-x_{0}+1}{2} \chi_{[\gamma, 1]},
$$


where

$$
\gamma=\frac{y_{0}-x_{0}+1}{\lambda} \in[0,1]
$$

On $[0, \gamma)$, the pair $\left(f_{1}, g_{1}\right)$ lies in $A_{\lambda}$, while on $[\gamma, 1]$ we have $\left(f_{1}, g_{1}\right)=\left(\left(x_{0}+y_{0}+1\right) / 2,\left(x_{0}+y_{0}-\right.\right.$ 1)/2) $\in B_{\lambda}$. Hence

$$
V_{\lambda}\left(x_{0}, y_{0}\right) \geq \gamma \cdot 1+(1-\gamma) \cdot \frac{1-x_{0}-y_{0}}{\lambda}=U_{\lambda}\left(x_{0}, y_{0}\right) .
$$

The case $2<\lambda<4$. For $\left(x_{0}, y_{0}\right) \in A_{\lambda}$ we prove (20) using the same processes as in the previous case, i.e. the constant ones if $y_{0} \geq \lambda$ and the ones given by (21) otherwise. The next step is to establish the inequality

$$
V_{\lambda}(-1, \lambda-2) \geq U_{\lambda}(-1, \lambda-2)=\frac{1+\alpha}{2}+\frac{1-\alpha}{2} \cdot\left(\frac{4-\lambda}{\lambda}\right)^{2} .
$$

To do this, fix $\delta \in(0,1]$ and set

$$
\beta=\frac{\delta(1-\alpha)}{\lambda}, \kappa=\frac{4-\lambda-\delta(1+\alpha)}{\lambda} \cdot \beta, \gamma=\beta+(1-\beta) \cdot \frac{\delta(1+\alpha)}{4}, v=\kappa \cdot \frac{\lambda}{4} .
$$

We have $0 \leq v \leq \kappa \leq \beta \leq \gamma \leq 1$. Consider processes $f$, $g$ given by $\left(f_{0}, g_{0}\right) \equiv(-1, \lambda-2)$, $\left(d f_{1}, d g_{1}\right) \equiv(\delta, \alpha \delta)$,

$$
\begin{gathered}
d f_{2}=-d g_{2}=\frac{\lambda-\delta(1-\alpha)}{2} \chi_{[0, \beta)}-\frac{\delta(1-\alpha)}{2} \chi_{[\beta, 1]}, \\
\left.d f_{3}=d g_{3}=-\left(\lambda-2+\frac{\delta(1+\alpha)}{2}\right) \chi_{[0, \kappa)}+\left(2-\frac{\lambda+\delta(1+\alpha)}{2}\right)\right) \chi_{[\kappa, \beta)} \\
+\left(2-\frac{\delta(1+\alpha)}{2}\right) \chi_{[\beta, \gamma)}-\frac{\delta(1+\alpha)}{2} \chi_{[\gamma, 1)}, \\
d f_{4}=-d g_{4}=\left(-2+\frac{\lambda}{2}\right) \chi_{[0, v)}+\frac{\lambda}{2} \chi_{[v, \kappa)} .
\end{gathered}
$$

As $\left(f_{4},\left|g_{4}\right|\right)$ takes values $(1, \lambda),(1,0)$ and $(-1, \lambda-2)$ with probabilities $(\gamma-\beta)+(\kappa-v), \beta-\kappa$ and $1-\gamma+v$, respectively, we have

$$
V_{\lambda}(-1, \lambda-2) \geq \gamma-\beta+\kappa-v+(1-\gamma+v) V_{\lambda}(-1, \lambda-2),
$$

or

$$
V_{\lambda}(-1, \lambda-2) \geq \frac{\gamma-\beta+\kappa-v}{\gamma-v}=\frac{1+\alpha}{2}+\frac{1-\alpha}{2} \cdot\left(\frac{4-\lambda}{\lambda}\right)^{2}-\frac{\delta\left(1-\alpha^{2}\right)}{\lambda^{2}} .
$$

As $\delta$ is arbitrary, we obtain (28). Now suppose $\left(x_{0}, y_{0}\right) \in B_{\lambda}$ and recall the pair $(f, g)$ starting from $\left(x_{0}, y_{0}\right)$ given by (22) and (23) (with $\beta$ defined in (24)). As previously, it leads to (25), which takes form

$$
\begin{aligned}
& V_{\lambda}\left(x_{0}, y_{0}\right) \geq \frac{\beta}{2}\left[\frac{1+\alpha}{2}+\frac{1-\alpha}{2} \cdot\left(\frac{4-\lambda}{\lambda}\right)^{2}\right]+1-\frac{\beta}{2} \\
& =\frac{\beta(1-\alpha)}{4}\left[\left(\frac{4-\lambda}{\lambda}\right)^{2}-1\right]+1=\frac{\left(\alpha x_{0}-\alpha-y_{0}+\lambda\right)(4-2 \lambda)}{\lambda^{2}}+1=U_{\lambda}\left(x_{0}, y_{0}\right) .
\end{aligned}
$$


For $\left(x_{0}, y_{0}\right) \in C_{\lambda}$, consider a pair $(f, g)$, starting from $\left(x_{0}, y_{0}\right)$ defined by (27) (with $\beta$ given by (26)). On $[0, \beta)$ we have $\left(f_{1}, g_{1}\right)=\left(\left(x_{0}+y_{0}+1-\lambda\right) / 2,\left(x_{0}+y_{0}-1+\lambda\right) / 2\right) \in B_{\lambda}$, so Lemma 4 yields

$$
\begin{aligned}
& V_{\lambda}\left(x_{0}, y_{0}\right) \geq \beta V_{\lambda}\left(\frac{x_{0}+y_{0}+1-\lambda}{2}, \frac{x_{0}+y_{0}-1+\lambda}{2}\right) \\
& =\frac{2\left(1-x_{0}\right)}{1+\lambda-x_{0}-y_{0}} \cdot\left\{1-\left[\alpha\left(\frac{x_{0}+y_{0}-1-\lambda}{2}\right)-\frac{x_{0}+y_{0}-1-\lambda}{2}\right] \cdot \frac{2 \lambda-4}{\lambda^{2}}\right\} \\
& =U_{\lambda}\left(x_{0}, y_{0}\right) .
\end{aligned}
$$

For $\left(x_{0}, y_{0}\right) \in D_{\lambda}$, set $\beta=\left(y_{0}-x_{0}+1\right) / \lambda \in[0,1]$ and let a pair $(f, g)$ be given by $\left(f_{0}, g_{0}\right) \equiv\left(x_{0}, y_{0}\right)$ and

$$
d f_{1}=-d g_{1}=\frac{-x_{0}+y_{0}+1-\lambda}{2} \chi_{[0, \beta)}+\frac{-x_{0}+y_{0}+1}{2} \chi_{[\beta, 1]} .
$$

As $\left(f_{1}, g_{1}\right)$ takes values

$$
\left(\frac{x_{0}+y_{0}+1-\lambda}{2}, \frac{x_{0}+y_{0}-1+\lambda}{2}\right) \in B_{\lambda} \text { and }\left(\frac{x_{0}+y_{0}+1}{2}, \frac{x_{0}+y_{0}-1}{2}\right) \in C_{\lambda}
$$

with probabilites $\beta$ and $1-\beta$, respectively, we obtain $V_{\lambda}\left(x_{0}, y_{0}\right)$ is not smaller than

$$
\begin{gathered}
\beta V_{\lambda}\left(\frac{x_{0}+y_{0}+1-\lambda}{2}, \frac{x_{0}+y_{0}-1+\lambda}{2}\right)+(1-\beta) V_{\lambda}\left(\frac{x_{0}+y_{0}+1}{2}, \frac{x_{0}+y_{0}-1}{2}\right) \\
=\frac{y_{0}-x_{0}+1}{\lambda} \cdot\left\{1-\left[\alpha\left(\frac{x_{0}+y_{0}-1-\lambda}{2}\right)-\frac{x_{0}+y_{0}-1-\lambda}{2}\right] \cdot \frac{2 \lambda-4}{\lambda^{2}}\right\} \\
+\frac{\lambda-y_{0}+x_{0}-1}{\lambda}\left[\frac{1-x_{0}-y_{0}}{\lambda}-\frac{\left(1-x_{0}-y_{0}\right)(1-\alpha)(\lambda-2)}{\lambda^{2}}\right] \\
=I+I I+I I I+I V,
\end{gathered}
$$

where

$$
I+I I I=\frac{y_{0}-x_{0}+1}{\lambda}+\frac{\left(\lambda-y_{0}+x_{0}-1\right)\left(1-x_{0}-y_{0}\right)}{\lambda^{2}}=\frac{2\left(1-x_{0}\right)}{\lambda}-\frac{\left(1-x_{0}\right)^{2}-y_{0}^{2}}{\lambda^{2}}
$$

and

$$
\begin{gathered}
I I+I V=\frac{(1-\alpha)(\lambda-2)}{\lambda^{3}}\left[\left(y_{0}-x_{0}+1\right)\left(y_{0}+x_{0}-1-\lambda\right)-\left(1-x_{0}-y_{0}\right)\left(\lambda-y_{0}+x_{0}-1\right)\right] \\
=-\frac{(1-\alpha)(\lambda-2)}{\lambda^{3}} \cdot \lambda\left(2-2 x_{0}\right) .
\end{gathered}
$$

Combining these facts, we obtain $V_{\lambda}\left(x_{0}, y_{0}\right) \geq U_{\lambda}\left(x_{0}, y_{0}\right)$.

For $\left(x_{0}, y_{0}\right) \in E_{\lambda}$ with $\left(x_{0}, y_{0}\right) \neq(-1,0)$, the following contruction will turn to be useful. Denote $w=\lambda-3$, so, as $\left(x_{0}, y_{0}\right) \in E_{\lambda}$, we have $x_{0}+y_{0}<w$. Fix positive integer $N$ and set $\delta=\delta_{N}=$ $\left(w-x_{0}-y_{0}\right) /[N(\alpha+1)]$. Consider sequences $\left(x_{j}^{N}\right)_{j=1}^{N+1},\left(p_{j}\right)_{j=1}^{N+1}$, defined by

$$
x_{j}^{N}=x_{0}+y_{0}+(j-1) \delta(\alpha+1), j=1,2, \ldots, N+1,
$$


and $p_{1}^{N}=\left(1+x_{0}\right) /\left(1+x_{0}+y_{0}\right)$,

$$
p_{j+1}^{N}=\frac{\left(1+x_{j}^{N}\right)\left(1+x_{j}^{N}+\frac{\delta(\alpha-1)}{2}\right) p_{j}^{N}}{\left(1+x_{j+1}^{N}\right)\left(1+x_{j}^{N}+\frac{\delta(\alpha+1)}{2}\right)}+\frac{\delta}{1+x_{j+1}^{N}}, j=1,2, \ldots, N .
$$

We construct a process $(f, g)$ starting from $\left(x_{0}, y_{0}\right)$ such that for $j=1,2, \ldots, N+1$,

$$
\begin{gathered}
\text { the variable }\left(f_{3 j},\left|g_{3 j}\right|\right) \text { takes values }\left(x_{j}^{N}, 0\right) \text { and }\left(-1,1+x_{j}^{N}\right) \\
\text { with probabilities } p_{j}^{N} \text { and } 1-p_{j}^{N} \text {, respectively. }
\end{gathered}
$$

We do this by induction. Let

$$
d f_{1}=-d g_{1}=y_{0} \chi_{\left[0, p_{1}^{N}\right)}+\left(-1-x_{0}\right) \chi_{\left[p_{1}^{N}, 1\right]}, d f_{2}=d g_{2}=d f_{3}=d g_{3}=0 .
$$

Note that (30) is satisfied for $j=1$. Now suppose we have a pair $(f, g)$, which satisfies (30) for $j=1,2, \ldots, n, n \leq N$. Let us describe $f_{k}$ and $g_{k}$ for $k=3 n+1,3 n+2,3 n+3$. The difference $d f_{3 n+1}$ is determined by the following three conditions: it is a martingale difference, i.e., satisfies $\mathbb{E}\left(d f_{3 n+1} \mid \mathscr{F}_{3 n}\right)=0$; conditionally on $\left\{f_{3 n}=x_{n}^{N}\right\}$, it takes values in $\left\{-1-x_{n}^{N}, \delta(\alpha+1) / 2\right\}$; and vanishes on $\left\{f_{3 n} \neq x_{n}^{N}\right\}$. Furthermore, set $d g_{3 n+1}=d f_{3 n+1}$. Moreover,

$$
d f_{3 n+2}=\delta \chi_{\left\{f_{3 n+1}=-1\right\}}, \quad d g_{3 n+2}=\frac{g_{3 n+1}}{\left|g_{3 n+1}\right|} \alpha \cdot d f_{3 n+2} .
$$

Finally, the variable $d f_{3 n+3}$ satisfies $\mathbb{E}\left(d f_{3 n+3} \mid \mathscr{F}_{3 n+2}\right)=0$, and, in addition, the variable $f_{3 n+3}$ takes values in $\left\{-1, x_{n}^{N}+\delta(\alpha+1)\right\}=\left\{-1, x_{n}^{N+1}\right\}$. The description is completed by

$$
d g_{3 n+3}=-\frac{g_{3 n+2}}{\left|g_{3 n+2}\right|} d f_{3 n+3} .
$$

One easily checks that $\left(f_{3 n+3},\left|g_{3 n+3}\right|\right)$ takes values in $\left\{\left(x_{n+1}^{N}, 0\right),\left(-1,1+x_{n+1}^{N}\right)\right\}$; moreover, since

$$
\begin{aligned}
\mathbb{E} f_{3 n+3} & =\mathbb{E} f_{3 n}+\mathbb{E} d f_{3 n+2}=x_{n}^{N} p_{n}^{N}-\left(1-p_{n}^{N}\right)+\delta \mathbb{P}\left(f_{3 n+1}=-1\right) \\
& =x_{n}^{N} p_{n}^{N}-\left(1-p_{n}^{N}\right)+\delta\left(1-p_{n}^{N}+p_{n}^{N} \frac{\delta(\alpha+1)}{2\left(1+x_{n}^{N}\right)+\delta(\alpha+1)}\right) \\
& =p_{n}^{N} \cdot \frac{\left(x_{n}^{N}+1\right)\left(1+x_{n}^{N}+\delta(\alpha-1) / 2\right)}{1+x_{n}^{N}+\delta(\alpha+1) / 2}+\delta-1,
\end{aligned}
$$

we see that $\mathbb{P}\left(f_{3 n+3}=x_{n+1}^{N}\right)=p_{n+1}^{N}$ and the pair $(f, g)$ satisfies (29) for $j=n+1$. Thus there exists $(f, g)$ satisfying $(29)$ for $j=1,2, \ldots, N+1$. In particular, $\left(f_{3(N+1)},\left|g_{3(N+1)}\right|\right)$ takes values $(w, 0),(-1, w+1) \in D_{\lambda}$ with probabilities $p_{N+1}^{N}, 1-p_{N+1}^{N}$. By Lemma 4 ,

$$
V_{\lambda}\left(x_{0}, y_{0}\right) \geq p_{N+1}^{N} V_{\lambda}(w, 0)+\left(1-p_{N+1}^{N}\right) V_{\lambda}(-1, w+1) .
$$

Recall the function $H$ defined by (2). The function $h:\left[x_{0}+y_{0}, w\right] \rightarrow \mathbb{R}$ given by $h(t)=$ $H\left(x_{0}, y_{0}, t\right)$, satisfies the differential equation

$$
h^{\prime}(t)+\frac{\alpha+2}{\alpha+1} \cdot \frac{h(t)}{1+t}=\frac{1}{(\alpha+1)(1+t)} .
$$


As we assumed $x_{0}+y_{0}>-1$, the expression $(h(x+\delta)-h(x)) / \delta$ converges uniformly to $h^{\prime}(x)$ on $\left[x_{0}+y_{0}, \lambda-3\right]$. Therefore there exist constants $\varepsilon_{N}$, which depend only on $N$ and $x_{0}+y_{0}$ satisfying $\lim _{N \rightarrow \infty} \varepsilon_{N}=0$ and for $1 \leq j \leq N$,

$$
\left|\frac{h\left(x_{j+1}^{N}\right)-h\left(x_{j}^{N}\right)}{(\alpha+1) \delta_{N}}+\frac{\left[\frac{\alpha+2}{\alpha+1}\left(1+x_{j}^{N}\right)-\frac{\delta_{N}(\alpha+1)}{2}\right] h\left(x_{j}^{N}\right)}{\left(1+x_{j+1}^{N}\right)\left(1+x_{j}^{N}+\frac{\delta_{N}(\alpha+1)}{2}\right)}-\frac{1}{(\alpha+1)\left(1+x_{j+1}^{N}\right)}\right| \leq \varepsilon_{N},
$$

or, equivalently,

$$
\left|h\left(x_{j+1}^{N}\right)-\frac{\left(1+x_{j}^{N}\right)\left(1+x_{j}^{N}+\frac{\delta_{N}(\alpha-1)}{2}\right) h\left(x_{j}^{N}\right)}{\left(1+x_{j+1}^{N}\right)\left(1+x_{j}^{N}+\frac{\delta_{N}(\alpha+1)}{2}\right)}-\frac{\delta_{N}}{1+x_{j+1}^{N}}\right| \leq(\alpha+1) \delta_{N} \varepsilon_{N} .
$$

Together with (29), this leads to

$$
\left|h\left(x_{j+1}^{N}\right)-p_{j+1}^{N}\right| \leq \frac{\left(1+x_{j}^{N}\right)\left(1+x_{j}^{N}+\frac{\delta_{N}(\alpha-1)}{2}\right)}{\left(1+x_{j+1}^{N}\right)\left(1+x_{j}^{N}+\frac{\delta_{N}(\alpha+1)}{2}\right)}\left|h\left(x_{j}^{N}\right)-p_{j}^{N}\right|+(\alpha+1) \delta_{N} \varepsilon_{N} .
$$

Since $p_{1}^{N}=h\left(x_{1}^{N}\right)$, we have

$$
\left|h(w)-p_{N+1}^{N}\right| \leq(\alpha+1) N \delta_{N} \varepsilon_{N}=\left(\lambda-3-x_{0}-y_{0}\right) \varepsilon_{N}
$$

and hence $\lim _{N \rightarrow \infty} p_{N+1}^{N}=h(w)$. Combining this with (31), we obtain

$$
V_{\lambda}\left(x_{0}, y_{0}\right) \geq h(w)\left(V_{\lambda}(w, 0)-V_{\lambda}(-1, w+1)\right)+V_{\lambda}(-1, w+1) .
$$

As $w=\lambda-3$, it suffices to check that we have

$$
\left.a_{\lambda}=V_{\lambda}(\lambda-3,0)-V_{\lambda}(-1, \lambda-2)\right) \text { and } b_{\lambda}=V_{\lambda}(-1, \lambda-2) \text {, }
$$

where $a_{\lambda}, b_{\lambda}$ were defined in (5). Finally, if $\left(x_{0}, y_{0}\right)=(-1,0)$, then considering a pair $(f, g)$ starting from $\left(x_{0}, y_{0}\right)$ and satisfying $d f_{1} \equiv \delta, d g_{1} \equiv \alpha \delta$, we get

$$
V(-1,0) \geq V(-1+\delta, \alpha \delta) .
$$

Now let $\delta \rightarrow 0$ to obtain $V(-1,0) \geq U(-1,0)$.

The case $\lambda \geq 4$. We proceed as in previous case. We deal with $\left(x_{0}, y_{0}\right) \in A_{\lambda}$ exactly in the same manner. Then we establish the analogue of (28), which is

$$
V(-1, \lambda-2) \geq U_{\lambda}(-1, \lambda-2)=\frac{1+\alpha}{2} .
$$

To do this, fix $\delta \in(0,1)$ and set

$$
\beta=\frac{4-2 \delta}{4-\delta(1+\alpha)}, \gamma=\beta \cdot\left(1-\frac{\delta(\alpha+1)}{4}\right) .
$$

Now let a pair $(f, g)$ be defined by $\left(f_{0}, g_{0}\right) \equiv(-1, \lambda-2),\left(d f_{1}, d g_{1}\right) \equiv(\delta, \alpha \delta)$,

$$
d f_{2}=-d g_{2}=-\frac{\delta(1-\alpha)}{2} \chi_{[0, \beta)}+(2-\delta) \chi_{[\beta, 1]},
$$




$$
d f_{3}=d g_{3}=-\frac{\delta(1+\alpha)}{2} \chi_{[0, \gamma)}+\left(2-\frac{\delta(1+\alpha)}{2}\right) \chi_{[\gamma, \beta)} .
$$

Then $\left(f_{3}, g_{3}\right)$ takes values $(-1, \lambda-2),(1, \lambda)$ and $(1, \lambda-4+\delta(\alpha+1))$ with probabilities $\gamma, \beta-\gamma$ and $1-\beta$, respectively, and Lemma 4 yields

$$
V(-1, \lambda-2) \geq \gamma V(-1, \lambda-2)+(\beta-\gamma) V(1, \lambda),
$$

or

$$
V(-1, \lambda-2) \geq \frac{\beta-\gamma}{1-\gamma}=\frac{(\alpha+1)(2-\delta)}{4-\delta(\alpha+1)} .
$$

It suffices to let $\delta \rightarrow 0$ to obtain (33). The cases $\left(x_{0}, y_{0}\right) \in B_{\lambda}, C_{\lambda}$ are dealt with using the same processes as in the case $\lambda \in(2,4)$. If $\left(x_{0}, y_{0}\right) \in D_{\lambda}$, then Lemma 4, applied to the pair $(f, g)$ given by $\left(f_{0}, g_{0}\right) \equiv\left(x_{0}, y_{0}\right), d f_{1}=-d g_{1}=-\left(1+x_{0}\right) \chi_{\left[0,\left(1-x_{0}\right) / 2\right)}+\left(1-x_{0}\right) \chi_{\left[\left(1-x_{0}\right) / 2,1\right]}$, yields

$$
V\left(x_{0}, y_{0}\right) \geq \frac{1-x_{0}}{2} V\left(-1, x_{0}+y_{0}+1\right) \text {. }
$$

Furthermore, for any number $y$ and any $\delta \in(0,1)$, we have

$$
V(-1, y) \geq V(-1+\delta, y+\alpha \delta)
$$

which is proved in the same manner as (32). Hence, for large $N$, if we set $\delta=\left(\lambda-3-x_{0}-\right.$ $\left.y_{0}\right) /(N(\alpha+1))$, the inequalities (34) and (35) give

$$
\begin{aligned}
V\left(x_{0}, y_{0}\right) \geq \frac{1-x_{0}}{2} & V\left(-1, x_{0}+y_{0}+1\right) \geq \frac{1-x_{0}}{2} V\left(-1+\delta, x_{0}+y_{0}+1+\alpha \delta\right) \\
& \geq \frac{1-x_{0}}{2}\left(1-\frac{\delta}{2}\right) V\left(-1, x_{0}+y_{0}+1+(\alpha+1) \delta\right) \\
& \geq \frac{1-x_{0}}{2}\left(1-\frac{\delta}{2}\right)^{N} V\left(-1, x_{0}+y_{0}+1+N(\alpha+1) \delta\right) \\
& =\frac{1-x_{0}}{2}\left(1-\frac{\lambda-3-x_{0}-y_{0}}{2 N(\alpha+1)}\right)^{N} V(-1, \lambda-2) \\
& =\frac{\left(1-x_{0}\right)(1+\alpha)}{4}\left(1-\frac{\lambda-3-x_{0}-y_{0}}{2 N(\alpha+1)}\right)^{N} .
\end{aligned}
$$

Now take $N \rightarrow \infty$ to obtain $V_{\lambda}\left(x_{0}, y_{0}\right) \geq U_{\lambda}\left(x_{0}, y_{0}\right)$.

Finally, if $\left(x_{0}, y_{0}\right) \in E_{\lambda}$ we use the pair $(f, g)$ used in the proof of the case $\left(x_{0}, y_{0}\right) \in E_{\lambda}, \lambda \in(2,4)$, with $\omega=1$. Then the process $(f,|g|)$ ends at the points $(1,0)$ and $(-1,2)$ with probabilities, which can be made arbitrarily close to $H\left(x_{0}, y_{0}, 1\right)$ and $1-H\left(x_{0}, y_{0}, 1\right)$, respectively. It suffices to apply Lemma 4 and check that it gives $V_{\lambda}\left(x_{0}, y_{0}\right) \geq U_{\lambda}\left(x_{0}, y_{0}\right)$.

Acknowledgement: The results were obtained while the author was visiting Université de FrancheComté in Besançon, France.

\section{References}

[1] D. L. Burkholder, Explorations in martingale theory and its applications, Ecole d'Ete de Probabilités de Saint-Flour XIX-1989, 1-66, Lecture Notes in Math., 1464, Springer, Berlin, 1991. MR1108183 
[2] D. L. Burkholder, Strong differential subordination and stochastic integration, Ann. Probab. 22 (1994), 995-1025. MR1288140

[3] C. Choi, A submartingale inequality, Proc. Amer. Math. Soc. 124 (1996), 2549-2553. MR1353381

[4] W. Hammack, Sharp inequalities for the distribution of a stochastic integral in which the integrator is a bounded submartingale, Ann. Probab. 23 (1995), 223-235. MR1330768 